\title{
Mobile Anti-phase Domains in Lightly Doped Lanthanum Cuprate
}

\author{
P. C. Hammel, B. J. Suh and J. L. Sarrao \\ Condensed Matter and Thermal Physics, Los Alamos National Laboratory, Los Alamos, NM 87545 \\ Z. Fisk \\ National High Magnetic Field Laboratory, Florida State University, Tallahassee, FL 32306
}

(Received: May 21, 1998)

\begin{abstract}
Light hole doping of lanthanum cuprate strongly suppresses the onset of antiferromagnetic (AF) order. Surprisingly, it simultaneously suppresses the extrapolated zero temperature sub-lattice magnetization. ${ }^{139} \mathrm{La} \mathrm{NQR}$ results in lightly doped $\mathrm{La}_{2} \mathrm{Cu}_{1-\mathrm{x}} \mathrm{Li}_{\mathrm{x}} \mathrm{O}_{4}$ have demonstrated that these effects are independent of the details of the mobility of the added holes. We propose a model in which doped holes phase separate into charged domain walls that surround "anti-phase" domains. These domains are mobile down to $\sim 30 \mathrm{~K}$ where they either become pinned to the lattice or evaporate as their constituent holes become pinned to dopant impurities.
\end{abstract}

PACS numbers: 75.30.Kz, 76.60.Jx, 74.72.Dn, 76.60.Gv

\section{INTRODUCTION}

A fundamental issue in the normal state of the superconducting cuprates is the behavior of holes doped into a two-dimensional lattice of spins with strong antiferromagnetic (AF) interactions. Even for lightly doped, single layer lanthanum cuprate many important issues remain poorly understood. Long-range antiferromagnetic order occurs at $T_{N}>300 \mathrm{~K}$ in undoped lanthanum cuprate, but $T_{N}$ is rapidly suppressed by the addition of a small density, $p$ of holes per $\mathrm{Cu}$. This rapid suppression is clearly related to the disruptive effects of mobile holes: $p \lesssim 3 \%$ is sufficient to suppress $T_{N}$ to zero, while $\sim 30 \%$ isovalent substitution of $\mathrm{Zn}$ or $\mathrm{Mg}$ for $\mathrm{Cu}$ is required [1] to produce the same effect. A range of studies [2] including ${ }^{139} \mathrm{La}$ NQR measurements [3] in lightly doped $\mathrm{La}_{2} \mathrm{Cu}_{1-\mathrm{x}} \mathrm{Li}_{\mathrm{x}} \mathrm{O}_{4}$ have demonstrated that the suppression of $T_{N}$, and in fact, all the magnetic properties of lightly doped lanthanum cuprate are essentially invariant without regard for the means of hole doping and consequent variations in hole mobility.

It is unlikely that a collection of individual holes can lead to magnetic behavior that is entirely independent of compositional variation that leads to substantial variations in resistivity (at constant doping). We argue, instead, that this is strong evidence that holes form collective structures. An important and well documented aspect of doped cuprates is their tendency toward inhomogeneous charge distribution [4]. Segregation of doped holes into charged stripes separating hole-free domains has been predicted 5 11 and recently observed directly in lanthanum cuprate [12]. It was proposed earlier that phase segregation of holes could be responsible for the unusual magnetic properties of lightly Sr-doped lanthanum cuprate 13 15]. We make a related proposal that holes form charged, domain walls which form closed loops with the important differences that these walls form anti-phase domain walls (so the the phase of the AF order inside these domains is reversed) and that the walls and hence the enclosed domains are mobile, and the charged walls have the density of 1 hole per $2 \mathrm{Cu}$ sites in agreement with neutron scattering results [12]. The anti-phase character means that mobile (above $30 \mathrm{~K}$ ) domains will suppress the time-averaged static moment thus suppressing $M_{s}$ as well as $T_{N}$. These domain structures will have contrasting interactions with in-plane vs. out-of-plane dopants (e.g., stronger scattering by in-plane impurities) which explain the different transport behaviors, while the universal magnetic properties can be understood as long as the domains are sufficiently mobile that they move across a given site rapidly compared to a measurement time.

\section{LIGHTLY DOPED LANTHANUM CUPRATE}

A systematic study of the temperature $T$ and doping dependence of the static susceptibility in lightly doped lanthanum cuprate by Cho et al. [13] provided evidence that the added holes are inhomogeneously distributed. The development of long-range antiferromagnetic order is signaled by a peak in the static susceptibility; they showed the rapid increase in the width of this peak with increasing hole density could be understood as arising from finite-size effects. They proposed that doped holes form hole-rich domain walls that bound hole-free domains, thus cutting off spin interactions across the boundary and truncating the growth of the spin-spin correlation length with decreasing temperature above $T_{N}$. They deduced the doping dependence of the dimension $\mathcal{L}$ of the hole free-regions, and found $\mathcal{L} \simeq(0.02 / p)^{2} ;$ this suggests that the density of holes within the boundary stripe is very low, $\sim 1$ hole per 5 or $10 \mathrm{Cu}$ sites.

${ }^{139} \mathrm{La}$ NQR measurements in lightly Sr-doped lanthanum cuprate by Chou et al. provided a detailed picture of the $T$ and $p$-dependence of its magnetic properties [14]. They found that for $30 \mathrm{~K} \lesssim T<T_{N}$ the sublattice 


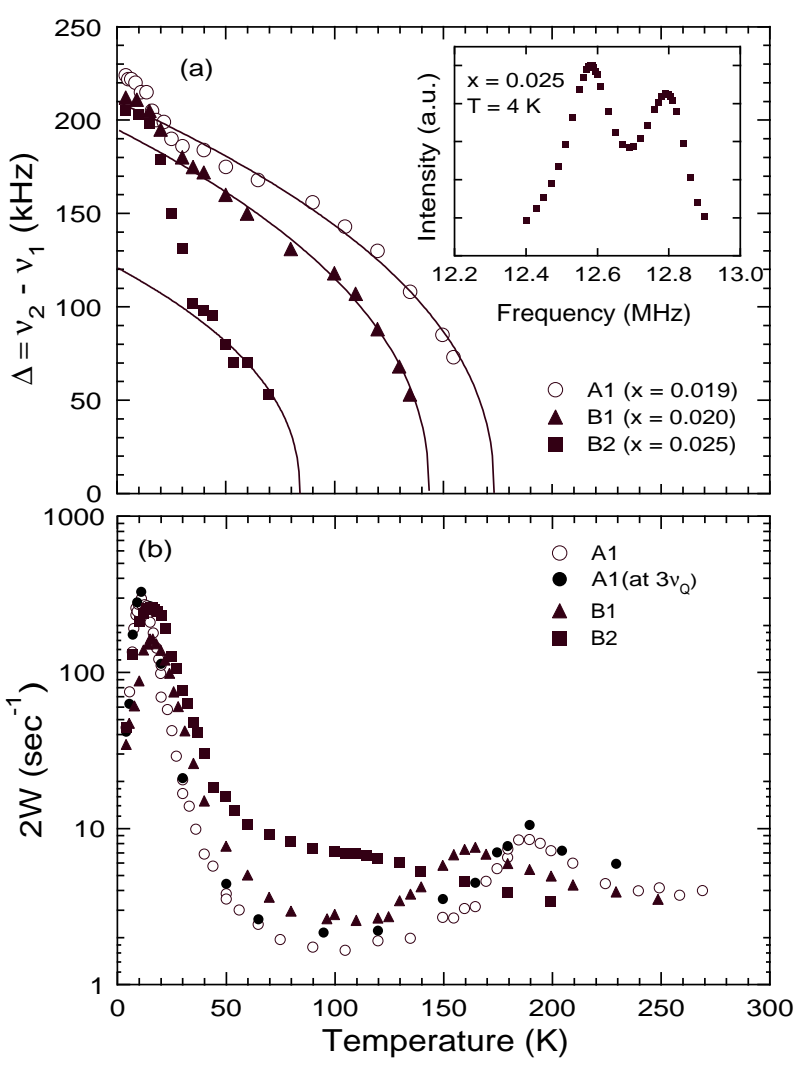

FIG. 1. ${ }^{139} \mathrm{La}$ NQR in $\mathrm{La}_{2} \mathrm{Cu}_{1-x} \mathrm{Li}_{x} \mathrm{O}_{4}:$ (a) $\Delta \equiv \nu_{1}-\nu_{2}$ vs. T. Solid curves are fits to the critical behavior $\Delta(T)=\Delta_{0}\left(1-T / T_{N}\right)^{\beta}$. The inset shows the split $2 \nu_{Q}$ transition at $4 \mathrm{~K}$ The magnitude of this splitting measures the component of the internal field due to the $\mathrm{AF}$ ordered $\mathrm{Cu}$ moments parallel to the EFG axis. (b) $2 W$ vs. $T$ is shown. The very strong peak in the vicinity of $15 \mathrm{~K}$ is very similar to that seen in Sr-doped lanthanum cuprate 14.

magnetization $M_{s}$ is strongly suppressed as $p$ increases. However below $30 \mathrm{~K}, M_{s}$ recovers to its $p=0$ value. The low temperature spin dynamics are also unusual; the ${ }^{139} \mathrm{La}$ nuclear spin-lattice relaxation rate $2 W\left(\equiv 1 / T_{1}\right)$ has a strong peak at a doping dependent temperature in the vicinity of $10-15 \mathrm{~K}$. To explain these unusual features, they extended the finite size model of Cho et al. [13], and proposed that the suppression of $M_{s}$ could be understood in the context of the restricted set of spinwave modes accessible in the confined AF domains [15. The low temperature peak in $2 W$ is clearly associated with freezing of $\mathrm{Cu}$ spin degrees of freedom; they interpreted this in terms of freezing out of hole motion within the domain walls surrounding hole-free regions.

Adding holes by means of in-plane substitution of $\mathrm{Li}^{1+}$ for $\mathrm{Cu}^{2+}$ introduces impurities into the $\mathrm{CuO}_{2}$ planes which strongly alter charge transport properties. We have used ${ }^{139} \mathrm{La}$ nuclear quadrupole resonance (NQR) measurements to microscopically examine the effects of doped holes on the AF spin correlations (Fig. 11) in this case where the hole mobility is much reduced compared

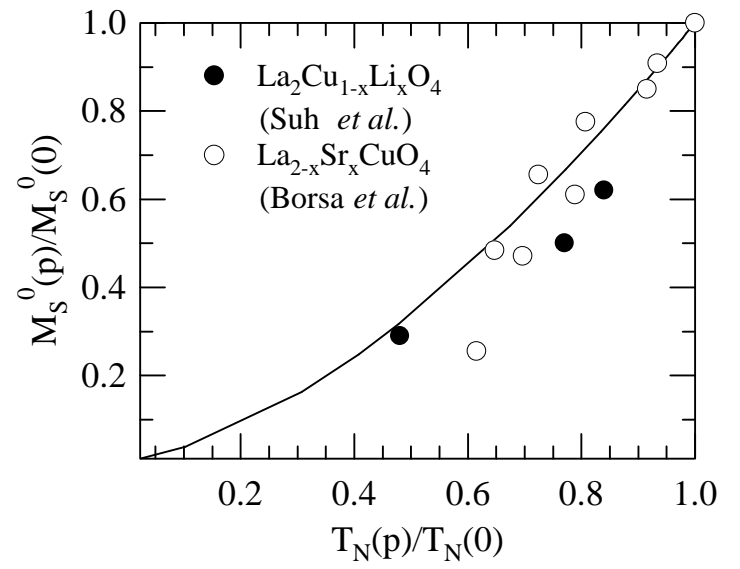

FIG. 2. The relationship between $M_{s}^{0}(p)$ and of $T_{N}(p)$ as both are suppressed by increasing doping $p . M_{s}^{0}(p)$ is obtained as explained in the text from ${ }^{139} \mathrm{La} \mathrm{NQR}$ data for $\Delta \nu$ such as is shown for the Li case in Fig. 1. The closed circles are from $\mathrm{La}_{2} \mathrm{Cu}_{1-x} \mathrm{Li}_{\mathrm{x}} \mathrm{O}_{4}$ [3], and the open circles are from $\mathrm{La}_{2-x} \mathrm{Sr}_{\mathrm{x}} \mathrm{CuO}_{4}$ 15. The results are the same in both materials, illustrating one aspect of the similarity of the magnetic properties, in spite of the much higher resistivity found in $\mathrm{La}_{2} \mathrm{Cu}_{1-\mathrm{x}} \mathrm{Li}_{\mathrm{x}} \mathrm{O}_{4}$ as a consequence of the in-plane impurities. The solid line is due to Castro Neto and Hone 16 .

to the $\mathrm{Sr}$ doping case [3]. Comparing $\mathrm{La}_{2-y} \mathrm{Sr}_{\mathrm{y}} \mathrm{CuO}_{4}$ (LSCO) and $\mathrm{La}_{2} \mathrm{Cu}_{1-\mathrm{x}} \mathrm{Li}_{\mathrm{x}} \mathrm{O}_{4}$ (LCLO) at $x=y=p=$ 0.025 one finds that the room temperature resistivity of LCLO 2, 17] exceeds that of LSCO [18] by over an order of magnitude. Furthermore, unlike LSCO, the resistivity of LCLO always increases monotonically with decreasing temperature. With increasing doping the contrast becomes more dramatic as LSCO becomes metallic and superconducting while LCLO becomes ever more insulating with doping above $p=0.1$.

In spite of this we find that the magnetic behavior of the two materials is essentially identical [3]. In addition to the similarly strong suppression of $T_{N}$ by doping [2], we find that $M_{s}$ is also suppressed, and the correspondence between the suppression of $M_{s}$ and $T_{N}$ by doping is identical to that observed in LSCO [15]. In Fig. 2 $M_{x}^{0}(p) / M_{x}^{0}(0)$ for both LCLO (Ref. [3]) and LSCO (Ref. [15]) is plotted against $T_{N}(p) / T_{N}(0)$. Here $M_{s}^{0}$ is the value of $M_{s}$ obtained by extrapolating the $M_{s}(T)$ data for $T>30 \mathrm{~K}$ to $T=0$ i.e., the $T=0$ value of the solid lines shown in Fig. 11(a). The solid line through the data is due to a theory of Castro Neto and Hone 16]; see also van Duin and Zaanen [19]. The strong peak in $2 W$ occurs at the same temperature and exhibits the same binding energy (as extracted from the $T$-dependence on the high temperature side of the peak) [3]. Finally, the temperature dependence of the low-energy dynamical susceptibility (obtained from measurements of $2 W(T)$ ) exhibits the same finite-size effects [3] as were observed in the static susceptibility by Cho et al [13]. 


\section{MOBILE ANTI-PHASE DOMAINS}

There is clear evidence for stripe formation in twodimensional doped antiferromagnets. In $\mathrm{La}_{2} \mathrm{NiO}_{4}$ (isostructural to lanthanum cuprate) static stripes have been observed in several cases 20 24. The recent observation of similar elastic superlattice peaks in $\mathrm{La}_{1.48} \mathrm{Nd}_{0.4} \mathrm{Sr}_{0.12} \mathrm{CuO}_{4}[12]$ demonstrates the existence of static charged stripes in the cuprates, and supports the idea that stripes are universally present in lanthanum cuprate 25.26] but that they are observable as static only under special conditions which pin the stripes to the lattice 12]. Similarities between elastic superlattice peaks associated with static stripes and the incommensurate peaks observed in inelastic neutron studies of $\mathrm{La}_{2-y} \mathrm{Sr}_{\mathrm{y}} \mathrm{CuO}_{4}$ 27] have been noted, and these incommensurate peaks are being reconsidered as possible evidence for the presence of dynamic charged stripes in the cuprate [28]. The density of holes in the charged domain walls depends on the material: in the nickelates it is 1 hole per stripe $\mathrm{Ni}$ site, and in the cuprate the density is $1 / 2$ hole per stripe $\mathrm{Cu}$ site. The neutron diffraction studies have demonstrated that spin-spin interactions are not cut off by the charged domain walls, rather interactions across them are strong: it is universally observed that they serve as anti-phase domain walls between the hole free regions they separate. Thus the sign of the spin correlations is reversed upon crossing the domain wall.

The formation of domain walls into loops as opposed to parallel stripes has been observed in Hartree-Fock calculations [5]. Using density matrix renormalization group techniques to calculate the energy of a domain wall in the 2D $t$ - $J$ model White and Scalapino have observed charged domain walls to form loops [29. They point out this is favorable at low doping in the case where the coupling between planes is significant. Because the walls constitute anti-phase domain walls, the coupling between two planes is disrupted, in general, by domain walls.

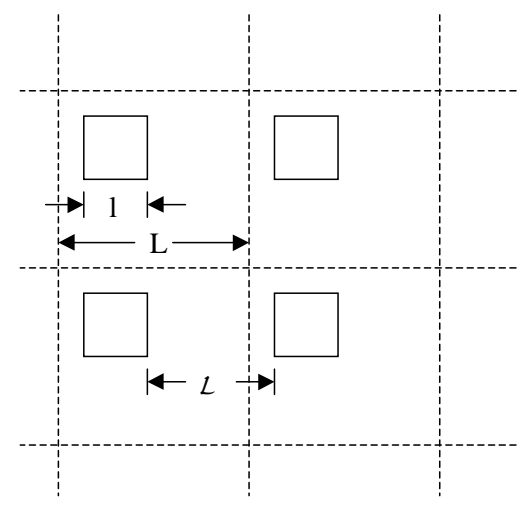

FIG. 3. Schematic diagram of the various lengths in the anti-phase domain model. On average, an area of dimension $L$ will contain a single anti-phase domain of dimension $l$. The length $\mathcal{L}=L-l$ is the finite-size confinement length that should be compared to the results obtained by Cho et al. [13.
Hence, inter-plane coupling would favor domain walls forming closed loops so that most of each plane would be in the dominant AF phase.

In the event that these anti-phase domains are mobile, passage of such a domain over a given site will reverse the orientation of a particular ordered $\mathrm{Cu}$ moment. The splitting of the ${ }^{139}$ La NQR line (shown in Fig. 1) is proportional to local hyperfine field due to the ordered moment on the neighboring $\mathrm{Cu}$ site. If this moment is time-varying, the splitting will be proportional to the time-averaged local moment. In the absence of anti-phase domains, the hyperfine field will be constant giving the value of $M_{s}$ observed in undoped lanthanum cuprate. If the motion of the anti-phase domains is rapid compared to the NQR measurement time, the net local hyperfine field will be proportional to the fraction of time the moment is in the dominant AF phase minus the time it is in an anti-phase domain, and hence proportional to the area of the dominant phase minus the area of the antiphase domain. We can estimate the doping dependence of the size and spacing of the anti-phase domains from the known behavior of $M_{s}^{0}$ in $\mathrm{La}_{2-y} \mathrm{Sr}_{\mathrm{y}} \mathrm{CuO}_{4}$ [15]. If we define $R(p) \equiv M_{s}^{0}(p) / M_{s}^{0}(0)$ the data [15] for $M_{s}^{0}(p)$ is well described by

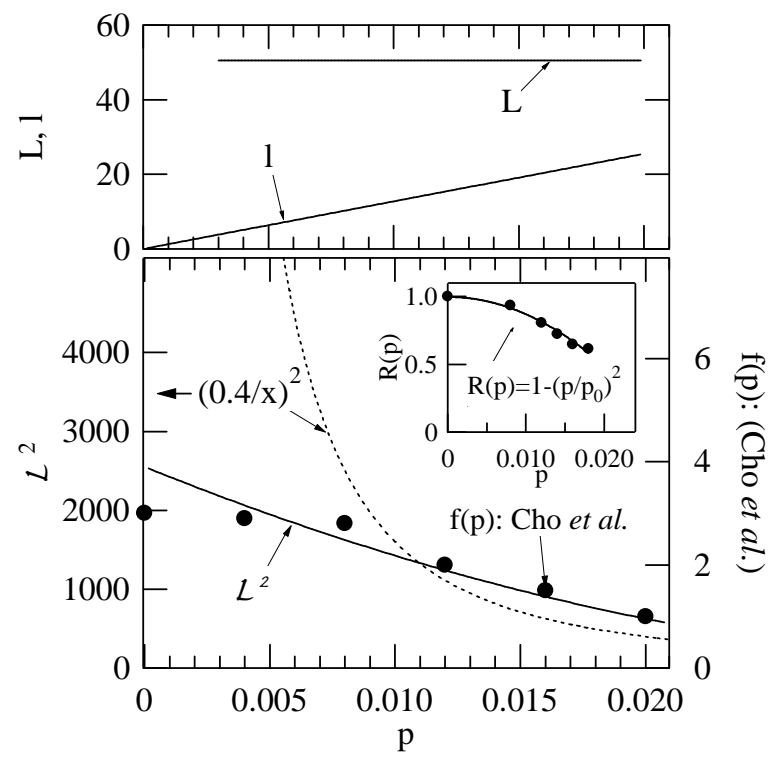

FIG. 4. Upper panel: The variation of the size $l$ of the anti-phase domains required to explain the observed suppression of $M_{s}^{0}$ by doping is shown along with the average size $L$ of the region which encompasses a single anti-phase domain. The inset to the lower panel shows the data of Borsa et al. for $R(p) \equiv M_{s}^{0}(p) / M_{s}^{0}(0)$ [15] along with the parametrization of the $p$-dependence used to calculate the lengths shown here; $p_{0}=0.028$. Lower panel: The variation of $\mathcal{L}^{2}$ with doping is plotted against the left-hand axis, and the results of Cho et al. 13. are plotted against the right-hand axis. The fit obtained by scaling the single parameter which sets the overall magnitude of $f(p)$ obtained by Cho et al. is very good. Also shown, plotted against the left-hand axis, is the fit $(0.4 / x)^{2}$ suggested by Cho et al. above $p=0.01$. 


$$
R(p)=1-\left(p / p_{0}\right)^{2}
$$

with $p_{0}=0.028$. For simplicity we assume that a $(1,0)$ or $(0,1)$ domain wall orientation is preferred, and so consider square domains.

If a region of size $L$ contains, on average, one antiphase domain of size $l$ (see Fig. 3, all lengths are in units of the lattice parameter), then

$$
R=1-\left(2 N_{-} / N\right)
$$

Here $N=N_{+}+N_{-}=L^{2}$, where $N_{-}=l^{2}$ is the number of sites in the anti-phase domain and $N_{+}$is the number of sites in the dominant AF phase. The number of holes in the region of size $L$ is $p L^{2}$; the domain wall which bounds the anti-phase domain contains 1 hole per $2 \mathrm{Cu}$ sites, so $4 l=2 p L^{2}$. From Eqns. 1 and 2

$$
L^{2}=N=2(1-R) / p^{2}=2 / p_{0}^{2}
$$

and

$$
l=(1-R) / p=p / p_{0}^{2}
$$

hence $L \simeq 50$. The variation of $l$ with $p$ based on the experimentally determined variation of $R(p)$ is shown in Fig. 1 (a). It should be noted that the behavior found here is particularly simple as a consequence of the parametrization of $R(p)$ chosen (Eq. 1); this parametrization is not uniquely determined by the data.

This simple model has several appealing features. The model described in Section II which relies on static domain walls implies a very low hole density in the wall ( 0.1-0.2 holes/Cu site) which must nonetheless maintain its integrity as a charged stripe and entirely cut off AF interactions across the stripe. Our model posits a density of 0.5 holes per $\mathrm{Cu}$ site such as is observed in neutron scattering and predicted by calculations [29]. The recovery of $M_{s}$ below $30 \mathrm{~K}$ is straightforwardly understandable since once motion of the anti-phase domains becomes slow compared to the NQR time scale $(\sim 0.1-1$ $\mu \mathrm{sec})$ time averaging of the reversed spin directions will cease and the full ordered moment will be observed. This could arise either from pinning of the anti-phase domain to the lattice or evaporation of the domain walls due pinning of the constituent holes to the charged donor impurities; in either case the coincidence of the recovery of $M_{s}$ and the freezing of spin degrees of freedom evidenced by the low $T$ peak in $2 W$ is naturally explained. The correspondence between suppression of $M_{s}^{0}$ and $T_{N}$ is natural in this case because interlayer coupling will be hampered wherever an anti-phase domain is present, thus impeding the development three-dimensional AF ordering. See the discussion in Ref. 29] in this regard.

This model also explains the finite-size effects revealed by the susceptibility analysis of Cho et al. [13] if we consider that the appropriate length scale between domain walls is $\mathcal{L}=(L-l)$. The variation of $\mathcal{L}^{2}$ with $p$ is shown in Fig. $4(b)$ and compared with the variation of the square of the characterisitic length scale obtained by Cho et al. [13. (scaled vertically to obtain the best agreement). Finally we note from Fig. $1(\mathrm{a})$, that $L$ and $l$ converge with increasing $p$, and we expect that loops will cease to be stable when $L$ approaches $l$. For the parametrization of $R(p)$ we have chosen, $L=l$ when $p=\sqrt{2} p_{0}=0.04$, near the doping at which the the metal-insulator transition and spin-glass behavior are found. We speculate, then, that these are related to the transition in the configuration of the charged domain walls from loops to parallel stripes.

In conclusion, we have presented a model which explains the range of unusual magnetic phenomena observed in lightly doped lanthanum cuprate. In particular, we can understand the insensitivity of magnetic properties to materials variations that substantially increase the resistivity. This indicates that mobile anti-phase domains play a central role in determining the magnetic properties of lightly doped lanthanum cuprate. It may point to an explanation of the poorly understood "spin-glass" regime of the phase diagram in terms of a crossover in domain wall topology from loops to parallel stripes. More generally, it suggests that the development of stripe order may play a determining role in the phase diagram of the cuprates (see e.g., Ref. [30]). Rather than requiring mobile domain walls, superconductivity may more sensitively depend on the nature of the ordering of the walls into parallel stripes.

\section{ACKNOWLEDGEMENTS}

We gratefully acknowledge stimulating conversations with J. Zaanen, who suggested the idea behind the model presented here. Work at Los Alamos performed under the auspices of the US Department of Energy. The NHMFL is supported by the NSF and the State of Florida through cooperative agreement DMR 95-27035.

[1] S. W. Cheong et al., Phys. Rev. B 44, 9739 (1991).

[2] J. L. Sarrao et al., Phys. Rev. B 54, 12014 (1996).

[3] B. J. Suh et al., cond-mat/9804200 (unpublished).

[4] Proceedings of the Workshop on Phase Separation in Cuprate Superconductors, edited by K. A. Müller and G. Benedek (World Scientific, Singapore, 1993).

[5] J. Zaanen and O. Gunnarsson, Phys. Rev. B 40, 7391 (1989).

[6] D. Poilblanc and T. M. Rice, Phys. Rev. B 39, 9749 (1989).

[7] H. J. Schulz, J. Phys. (Paris) 50, 2833 (1989).

[8] V. J. Emery, S. A. Kivelson, and H. Q. Lin, Phys. Rev. Lett. 64, 475 (1990). 
[9] H. E. Viertiö and T. M. Rice, J. Phys. Condens. Matter 6, 7091 (1994).

[10] M. Kato, K. Machida, H. Nakanish, and M. Fujita, J. Phys. Soc. Jpn. 59, 1047 (1990).

[11] J. Zaanen, cond-mat/9711009 (unpublished).

[12] J. M. Tranquada et al., Nature 375, 561 (1995).

[13] J. H. Cho, F. C. Chou, and D. C. Johnston, Phys. Rev. Lett. 70, 222 (1993).

[14] F. C. Chou et al., Phys. Rev. Lett. 71, 2323 (1993).

[15] F. Borsa et al., Phys. Rev. B 52, 7334 (1995).

[16] A. H. C. Neto and D. Hone, Phys. Rev. Lett. 76, 2165 (1996).

[17] M. A. Kastner et al., Phys. Rev. B 37, 111 (1988).

[18] H. Takagi et al., in Proceedings of the Workshop on Phase Separation in Cuprate Superconductors, edited by K. A. Müller and G. Benedek (World Scientific, Singapore, 1993), pp. 165-176. See Ref. 《4.

[19] C. N. A. vanDuin and J. Zaanen, Phys. Rev. Lett. 78, 3019 (1997).

[20] S. M. Hayden et al., Phys. Rev. Lett. 68, 1061 (1992).

[21] C. H. Chen, S.-W. Cheong, and A. S. Cooper, Phys. Rev. Lett. 71, 2187 (1993).

[22] J. M. Tranquada, D. J. Buttrey, V. Sachan, and J. E. Lorenzo, Phys. Rev. Lett. 73, 1003 (1994).

[23] J. M. Tranquada, D. J. Buttrey, and V. Sachan, Phys. Rev. B 54, 12318 (1996).

[24] S.-H. Lee and S. Cheong, Phys. Rev. Lett. 79, 2514 (1997).

[25] V. J. Emery and S. A. Kivelson, Phys. Rev. Lett. 74, 3253 (1995).

[26] J. Zaanen, M. L. Horbach, and W. Vansaarloos, Phys. Rev. B 53, 8671 (1996).

[27] S. W. Cheong et al., Phys. Rev. Lett. 67, 1791 (1991).

[28] J. M. Tranquada, Physica C 282, 166 (1997).

[29] S. R. White and D. J. Scalapino, cond-mat/9801274 (unpublished).

[30] S. A. Kivelson, E. Fradkin, and V. Emery, condmat/9707327 (unpublished). 